\title{
Quasilinear Schrödinger equations, Il: Small data and cubic nonlinearities
}

\author{
Jeremy L. Marzuola, Jason Metcalfe, and Daniel Tataru
}

\begin{abstract}
In part I of this project we examined low-regularity local well-posedness for generic quasilinear Schrödinger equations with small data. This improved, in the small data regime, the preceding results of Kenig, Ponce, and Vega as well as Kenig, Ponce, Rolvung, and Vega. In the setting of quadratic interactions, the (translation invariant) function spaces which were utilized incorporated an $l^{1}$-summability over cubes in order to account for Mizohata's integrability condition, which is a necessary condition for the $L^{2}$ well-posedness for the linearized equation. For cubic interactions, this integrability condition meshes better with the inherent $L^{2}$-nature of the Schrödinger equation, and such summability is not required. Thus we are able to prove small data well-posedness in $H^{s}$-spaces.
\end{abstract}

\section{Introduction}

We shall examine local well-posedness for quasilinear Schrödinger equations with cubic interactions and a Cauchy datum in a low-regularity Sobolev space. In particular, we examine

$$
\left\{\begin{array}{l}
i \partial_{t} u+g^{j k}(u, \nabla u) \partial_{j} \partial_{k} u=F(u, \nabla u), \quad u: \mathbb{R} \times \mathbb{R}^{d} \rightarrow \mathbb{C}^{m} \\
u(0, x)=u_{0}(x)
\end{array}\right.
$$

where

$$
g: \mathbb{C}^{m} \times\left(\mathbb{C}^{m}\right)^{d} \rightarrow \mathbb{R}^{d \times d}, \quad F: \mathbb{C}^{m} \times\left(\mathbb{C}^{m}\right)^{d} \rightarrow \mathbb{C}^{m}
$$

are smooth functions which satisfy

$$
g(y, z)=I_{d}+O\left(|y|^{2}+|z|^{2}\right), \quad F(y, z)=O\left(|y|^{3}+|z|^{3}\right) \quad \text { near }(y, z)=(0,0) .
$$

We also examine

$$
\left\{\begin{array}{l}
i \partial_{t} u+\partial_{j} g^{j k}(u) \partial_{k} u=F(u, \nabla u), \quad u: \mathbb{R} \times \mathbb{R}^{d} \rightarrow \mathbb{C}^{m}, \\
u(0, x)=u_{0}(x)
\end{array}\right.
$$

Kyoto Journal of Mathematics, Vol. 54, No. 3 (2014), 529-546 DOI $10.1215 / 21562261-2693424$, (C) 2014 by Kyoto University

Received October 4, 2012. Revised May 30, 2013. Accepted May 30, 2013.

2010 Mathematics Subject Classification: Primary 35Q55; Secondary 35A01, 35B30.

Metcalfe's work supported in part by National Science Foundation grant DMS-1054289.

Tataru's work supported in part by National Science Foundation grant DMS-0801261 as well as by the Miller Foundation. 
where $g$ depends only on $u$. The latter class of equations can be obtained by differentiating the first equation. Indeed, for $u$ solving (1.1), the vector $(u, \nabla u)$ solves an equation of the latter type. The latter is written in divergence form, which follows easily for this class of equations as the terms obtained when commuting $g$ with the derivative can be absorbed into $F(u, \nabla u)$.

The case of small initial data and quadratic, rather than cubic, nonlinear terms, was considered in [10]. There, local well-posedness was established for data of Sobolev-type regularity $s>\frac{d}{2}+3$ for (1.1) and $s>\frac{d}{2}+2$ for (1.2). This represented a significant improvement in regularity over the previous results (see [7], [3], [4]), though data of arbitrary size was examined there. A more complete history of such problems can be found in [10], [7], and [8].

In the quadratic case above, it is insufficient, however, to simply work in Sobolev spaces. Indeed, one encounters Mizohata's integrability condition (see [11]-[13], [2], [9]), which says that for the linear equation

$$
\left(i \partial_{t}+\Delta_{g}\right) v=A_{i}(x) \partial_{i} v,
$$

a necessary condition for $L^{2}$ well-posedness is an integrability of the real part of $A$ along the Hamiltonian flow of the leading-order operator. The potentials encountered when linearizing, for example, (1.1) with quadratic interactions do not necessarily satisfy such a condition even with arbitrarily high regularities. For this reason, the initial data spaces need to incorporate some decay. The translation-invariant approach of [10] was to require a summability over cubes, which was inspired by the earlier work [1] on semilinear derivative Schrödinger equations.

In the case of only cubic and higher-order interactions, the scenario is much simpler, which is what we shall demonstrate here. When linearizing (1.1) as stated, the potential that is encountered is $O\left(|u|^{2}+|\nabla u|^{2}\right)$, for which integrability follows easily from energy estimates. As such, the additional $l^{1}$-summability which was previously required is no longer needed, and the initial datum can be simply taken to be a member of a Sobolev space. This notion was previously explored in [5] and [6], and we seek to improve on that by considering rough initial data.

Our main result is precisely that (1.1) and (1.2) are locally well-posed for $u_{0}(x) \in H^{s}$.

\section{THEOREM 1}

(a) Let $s>\frac{d+5}{2}$. Then there exists $\epsilon>0$ sufficiently small such that for all initial data $u_{0}$ with

$$
\left\|u_{0}\right\|_{H^{s}} \leq \epsilon
$$

the (1.1) is locally well-posed in $H^{s}\left(\mathbb{R}^{d}\right)$ on the time interval $I=[0,1]$.

(b) The same result holds for the (1.2) with $s>\frac{d+3}{2}$.

In the theorem, well-posedness is taken to include the existence of a local solution, uniqueness, and continuous dependence on the initial datum. 
The above theorem also holds for ultrahyperbolic operators, as in [3] and [4], where $g(0)$ is of a different signature. Our method of proof of the local smoothing estimates uses a wedge decomposition, and the estimates are proved in each coordinate direction separately. Thus, trivial adjustments of the sign of the multiplier in the corresponding directions of opposite signature will permit said results.

This article is organized as follows. In Section 2, we set our notation and describe the function spaces in which we shall work. Section 3 is devoted to proving the required nonlinear estimates. Section 4 contains the proof of our main linear estimate, which is a variant of the classical local smoothing estimate for the Schrödinger equation. In Section 5 we prove Theorem 1.

\section{Function spaces and notation}

The function spaces and notation presented in this section are the same as those established in [10]. We repeat them for easy reference.

For a function $u(t, x)$ or $u(x)$, we let $\hat{u}=\mathcal{F} u$ denote the Fourier transform in the spatial variable $x$. A function $u$ is said to be localized at frequency $2^{i}$ if $\hat{u}(t, \xi)$ is supported in $\mathbb{R} \times\left\{\xi \in \mathbb{R}^{d}:|\xi| \in\left[2^{i-1}, 2^{i+1}\right]\right\}$. We shall use a Littlewood-Paley decomposition of the spatial frequencies,

$$
\sum_{i=0}^{\infty} S_{i}(D)=1
$$

where $S_{i}$ localizes to frequency $2^{i}$ for $i>0$ and to frequencies $|\xi| \leq 2$ for $i=0$. Note that we are working in nonhomogeneous spaces. We set

$$
u_{i}=S_{i} u, \quad S_{\leq N} u=\sum_{i=0}^{N} S_{i} u, \quad S_{\geq N} u=\sum_{i=N}^{\infty} S_{i} u .
$$

For each $j \in \mathbb{N}$, we let $\mathcal{Q}_{j}$ denote a partition of $\mathbb{R}^{d}$ into cubes of side length $2^{j}$, and we let $\left\{\chi_{Q}\right\}$ denote an associated partition of unity. For a translationinvariant Sobolev-type space $U$, we set $l_{j}^{p} U$ to be the Banach space with associated norm

$$
\|u\|_{l_{j}^{p} U}^{p}=\sum_{Q \in \mathcal{Q}_{j}}\left\|\chi_{Q} u\right\|_{U}^{p}
$$

with the obvious modification for $p=\infty$.

Within such norms, the smooth partition of unity with compactly supported functions $\chi_{Q}$ can be replaced by cutoffs which are frequency localized as the associated Schwartz tails decay rapidly away from $Q$. Thus, the components can be taken to retain the frequency localization of the function $u$.

Motivated by the well-known local smoothing estimates, we define

$$
\|u\|_{X}=\sup _{l} \sup _{Q \in \mathcal{Q}_{l}} 2^{-\frac{l}{2}}\|u\|_{L^{2} L^{2}([0,1] \times Q)} .
$$

Here and throughout, $L^{p} L^{q}$ represents $L_{t}^{p} L_{x}^{q}$. 
To measure the forcing terms, we use an atomic space $Y$ satisfying $X=Y^{*}$ (see [10]). A function $a$ is an atom in $Y$ if there is a $j \geq 0$ and a $Q \in \mathcal{Q}_{j}$ such that $a$ is supported in $[0,1] \times Q$ and

$$
\|a\|_{L^{2}([0,1] \times Q)} \lesssim 2^{-\frac{j}{2}} .
$$

Then we define $Y$ as linear combinations of the form

$$
f=\sum_{k} c_{k} a_{k}, \quad \sum\left|c_{k}\right|<\infty, a_{k} \text { atoms, }
$$

with norm

$$
\|f\|_{Y}=\inf \left\{\sum\left|c_{k}\right|: f=\sum_{k} c_{k} a_{k}, a_{k} \text { atoms }\right\}
$$

For solutions which are localized to frequency $2^{j}$, we shall incorporate the typical half-derivative smoothing by working within

$$
X_{j}=2^{-\frac{j}{2}} X \cap L^{\infty} L^{2}
$$

with norm

$$
\|u\|_{X_{j}}=2^{\frac{j}{2}}\|u\|_{X}+\|u\|_{L^{\infty} L^{2}} .
$$

We incorporate the Sobolev regularity and the cube summability by defining

$$
\|u\|_{l^{p} X^{s}}^{2}=\sum_{j \geq 0} 2^{2 j s}\left\|S_{j} u\right\|_{l_{j}^{p} X_{j}}^{2} .
$$

For the purposes of this paper, we will be working primarily in $l^{2} X^{s}$, as opposed to our previous paper [10] where we had to work in $l^{1} X^{s}$ due to incompatibilities with the Mizohata condition. On occasion we will also use the slightly larger space

$$
\|u\|_{X^{s}}^{2}=\sum_{j} 2^{2 j s}\left\|S_{j} u\right\|_{X_{j}}^{2}
$$

We analogously define

$$
Y_{j}=2^{\frac{j}{2}} Y+L^{1} L^{2}
$$

which has norm

$$
\|f\|_{Y_{j}}=\inf _{f=2^{\frac{j}{2}} f_{1}+f_{2}}\left\|f_{1}\right\|_{Y}+\left\|f_{2}\right\|_{L^{1} L^{2}}
$$

and

$$
\|f\|_{l^{p} Y^{s}}^{2}=\sum_{j} 2^{2 j s}\left\|S_{j} f\right\|_{l_{j}^{p} Y_{j}}^{2} .
$$

Here, we shall be working primarily within $l^{2} Y^{s}$, though we note that the Minkowski integral inequality gives

$$
\|f\|_{l^{2} Y^{s}} \lesssim\|f\|_{Y^{s}}
$$

where 


$$
\|f\|_{Y^{s}}^{2}=\sum_{j} 2^{2 j s}\left\|S_{j} f\right\|_{Y_{j}}^{2} .
$$

Hence, the cube summation will largely be ignored for the forcing terms.

We also note that for any $j$, we have

$$
\sup _{Q \in \mathcal{Q}_{j}} 2^{-\frac{j}{2}}\|u\|_{L^{2} L^{2}([0,1] \times Q)} \leq\|u\|_{X}
$$

hence

$$
\|u\|_{Y} \lesssim 2^{\frac{j}{2}}\|u\|_{l_{j}^{1} L^{2} L^{2}}
$$

This bound will come in handy at several places later on.

In our multilinear estimates, we shall use frequency envelopes. Consider a Sobolev-type space $U$ for which we have

$$
\|u\|_{U}^{2} \sim \sum_{k=0}^{\infty}\left\|S_{k} u\right\|_{U}^{2} .
$$

A frequency envelope for a function $u \in U$ is a positive $l^{2}$-sequence, $\left\{a_{j}\right\}$, with

$$
\left\|S_{j} u\right\|_{U} \leq a_{j}\|u\|_{U} .
$$

We shall only permit slowly varying frequency envelopes. Thus, we require $a_{0} \approx 1$ and

$$
a_{j} \leq 2^{\delta|j-k|} a_{k}, \quad j, k \geq 0,0<\delta \ll 1 .
$$

The constant $\delta$ shall be chosen later and only depends on $s$ and the dimension $d$. Such frequency envelopes always exist. For example, one may choose

$$
a_{j}=2^{-\delta j}+\|u\|_{U}^{-1} \sup _{k} 2^{-\delta|j-k|}\left\|S_{k} u\right\|_{U} .
$$

\section{Multilinear and nonlinear estimates}

This section contains our main multilinear estimates. The first proposition is essentially from [9], though it is somewhat simpler as the summability over cubes is easier.

\section{PROPOSITION 3.1}

Let $\sigma>\frac{d}{2}$, and let $u, v \in l^{2} X^{\sigma}$ with admissible frequency envelopes $a_{k}, b_{k}$, respectively. Then the $l^{2} X^{\sigma}$-spaces satisfy the algebra-type property

$$
\left\|S_{k}(u v)\right\|_{l^{2} X^{\sigma}} \lesssim\left(a_{k}+b_{k}\right)\|u\|_{l^{2} X^{\sigma}}\|v\|_{l^{2} X^{\sigma}},
$$

as well as the Moser-type estimate

$$
\left\|S_{k} F(u)\right\|_{l^{2} X^{\sigma}} \lesssim a_{k}\|u\|_{l^{2} X^{\sigma}}\left(1+\|u\|_{l^{2} X^{\sigma}}\right) c\left(\|u\|_{L^{\infty}}\right),
$$

for all smooth $F$ with $F(0)=0$.

Proof

To prove (3.1), we consider $\left\|S_{k}\left(S_{i} u S_{j} v\right)\right\|_{l_{k}^{2} X_{k}}$. We must have either $i \geq k-4$ or $j \geq k-4$; by symmetry we shall assume the former. Since $i \geq k-4$, we have 
$X_{i} \subset X_{k}$. And we naively use the fact that there are nearly equal to $2^{d(i-k)}$ cubes of side length $2^{k}$ contained in one of side length $2^{i}$. Thus, by Bernstein's inequality, we have

$$
\begin{aligned}
\left\|S_{k}\left(S_{i} u S_{j} v\right)\right\|_{l_{k}^{2} X_{k}} & \lesssim 2^{\frac{d}{2}(i-k)}\left\|S_{i} u\right\|_{l_{i}^{2} X_{i}}\left\|S_{j} v\right\|_{L^{\infty}} \\
& \lesssim 2^{\frac{d}{2}(i-k)} 2^{j \frac{d}{2}}\left\|S_{i} u\right\|_{l_{i}^{2} X_{i}}\left\|S_{j} v\right\|_{l_{j}^{2} L^{\infty} L^{2}}
\end{aligned}
$$

It follows that

$$
\left\|S_{k}\left(S_{i} u S_{j} v\right)\right\|_{l^{2} X^{\sigma}} \lesssim a_{i} b_{j} 2^{i\left(\frac{d}{2}-\sigma\right)} 2^{j\left(\frac{d}{2}-\sigma\right)} 2^{k\left(\sigma-\frac{d}{2}\right)}\|u\|_{l^{2} X^{\sigma}}\|v\|_{l^{2} X^{\sigma}} .
$$

As $\sigma>\frac{d}{2}$, the estimate (3.1) now follows after summation in $i, j$. When $k-4 \leq$ $i \leq k+4$, we simply use the fact that the Cauchy-Schwarz inequality implies that $\sum_{j} 2^{j\left(\frac{d}{2}-\sigma\right)} b_{j}$ is $O(1)$. When $i>k+4$, we also use $\sum_{i>k} 2^{i\left(\frac{d}{2}-\sigma\right)} a_{i} \lesssim 2^{k\left(\frac{d}{2}-\sigma\right)} a_{k}$ provided the $\delta$ in (2.2) satisfies $0<\delta<\sigma-\frac{d}{2}$.

We now examine (3.2) and replace the discrete Littlewood-Paley decomposition by a continuous one,

$$
\mathrm{Id}=S_{0}+\int_{0}^{\infty} S_{k} d k .
$$

Abusing notation, we will set $u_{0}=S_{0} u$ for now. Then, using the fundamental theorem of calculus, we can write

$$
S_{k} F(u)=S_{k} F\left(u_{0}\right)+\int_{0}^{\infty} S_{k}\left(u_{k_{1}} F^{\prime}\left(u_{<k_{1}}\right)\right) d k_{1} .
$$

For the first term of (3.4), we note that

$$
\left\|\partial^{\alpha} F\left(u_{0}\right)\right\|_{l_{0}^{2} X_{0}} \lesssim\left\|u_{0}\right\|_{l_{0}^{2} X_{0}} c\left(\left\|u_{0}\right\|_{L^{\infty}}\right)
$$

Thus,

$$
\left\|S_{k} F\left(u_{0}\right)\right\|_{l_{k}^{2} X_{k}} \lesssim 2^{k / 2}\left\|S_{k} F\left(u_{0}\right)\right\|_{l_{0}^{2} X_{0}} \lesssim 2^{-N k}\left\|u_{0}\right\|_{l_{0}^{2} X_{0}} c\left(\left\|u_{0}\right\|_{L^{\infty}}\right)
$$

for any $N$, from which the $l^{2} X^{\sigma}$-bound follows easily.

We split the integral in the second term of (3.4) into two regions. For $k_{1} \geq k-4$, we use that $X_{k_{1}} \subset X_{k}$, and we have

$$
\begin{aligned}
& 2^{k \sigma}\left\|\int_{k-4}^{\infty} S_{k}\left(u_{k_{1}} F^{\prime}\left(u_{<k_{1}}\right)\right) d k_{1}\right\|_{l_{k}^{2} X_{k}} \\
& \quad \lesssim \int_{k-4}^{\infty} 2^{-\left(k_{1}-k\right)\left(\sigma-\frac{d}{2}\right)}\left\|u_{k_{1}}\right\|_{l^{2} X^{\sigma}}\left\|F^{\prime}\left(u_{<k_{1}}\right)\right\|_{L^{\infty}} d k_{1}
\end{aligned}
$$

by using (3.3). From this, the desired bound follows if $\delta$ in (2.2) is chosen small enough. This case is significantly simpler here than its analogous case in [10] because we are now dealing with $l^{2}$-sums rather than $l^{1}$-sums.

For $k_{1}<k-4$, we note that

$$
S_{k}\left(u_{k_{1}} F^{\prime}\left(u_{<k_{1}}\right)\right)=S_{k}\left(u_{k_{1}} \tilde{S}_{k} F^{\prime}\left(u_{<k_{1}}\right)\right),
$$


where $\tilde{S}_{k}$ also localizes to frequency $2^{k}$ and $S_{k} \tilde{S}_{k}=S_{k}$. The chain rule allows us to estimate

$$
\left\|\tilde{S}_{k} F^{\prime}\left(u_{<k_{1}}\right)\right\|_{L^{\infty}} \lesssim 2^{-N\left(k-k_{1}\right)} c\left(\left\|u_{<k_{1}}\right\|_{L^{\infty}}\right)
$$

Thus,

$$
\begin{aligned}
\left\|S_{k}\left(u_{k_{1}} F^{\prime}\left(u_{<k_{1}}\right)\right)\right\|_{l_{k}^{2} X_{k}} & \lesssim 2^{\frac{k-k_{1}}{2}}\left\|u_{k_{1}}\right\|_{l_{k_{1}}^{2} X_{k_{1}}}\left\|\tilde{S}_{k} F^{\prime}\left(u_{<k_{1}}\right)\right\|_{L^{\infty}} \\
& \lesssim 2^{-N\left(k-k_{1}\right)}\left\|u_{k_{1}}\right\|_{l_{k_{1}}^{2} X_{k_{1}}} c\left(\left\|u_{<k_{1}}\right\|_{L^{\infty}}\right) .
\end{aligned}
$$

In turn, this leads to

$$
\left\|S_{k}\left(u_{k_{1}} F^{\prime}\left(u_{<k_{1}}\right)\right)\right\|_{l^{2} X^{\sigma}} \lesssim 2^{-N\left(k-k_{1}\right)} a_{k_{1}}\|u\|_{l^{2} X^{\sigma}} c\left(\|u\|_{L^{\infty}}\right),
$$

where as $N$ was arbitrary, we allowed its value to change from line to line, and after integration, (3.2) is proved.

Replacing the bilinear estimates which were used in [10] are the following trilinear estimates. In particular, we note that here we only require $s>\frac{d+3}{2}$, which accounts for the improvement in regularity as compared to the quadratic interactions explored in [10].

\section{PROPOSITION 3.2}

Let $s>\frac{d+3}{2}$, and suppose that $u \in l^{2} X^{\sigma-1}, v, w \in l^{2} X^{s-1}$ with frequency envelopes $\left\{a_{k}\right\},\left\{b_{k}\right\}$, and $\left\{c_{k}\right\}$, respectively. Then for $0 \leq \sigma \leq s$, we have

$$
\left\|S_{k}(u v w)\right\|_{l^{2} Y^{\sigma}} \lesssim\left(a_{k}+b_{k}+c_{k}\right)\|u\|_{l^{2} X^{\sigma-1}}\|v\|_{l^{2} X^{s-1}}\|w\|_{l^{2} X^{s-1}} .
$$

If $0 \leq \sigma \leq s-1$ and if $u \in l^{2} X^{\sigma}, v \in l^{2} X^{s-2}$, and $w \in l^{2} X^{s-1}$, then

$$
\left\|S_{k}(u v w)\right\|_{l^{2} Y^{\sigma}} \lesssim\left(a_{k}+b_{k}+c_{k}\right)\|u\|_{l^{2} X^{\sigma}}\|v\|_{l^{2} X^{s-2}}\|w\|_{l^{2} X^{s-1}} .
$$

Finally, for $0 \leq \sigma \leq s$ and $u \in l^{2} X^{\sigma-2}$ and $v, w \in l^{2} X^{s}$,

$$
\left\|S_{k}\left(u S_{\geq k-4}(v w)\right)\right\|_{l^{2} Y^{\sigma}} \lesssim\left(a_{k}+b_{k}+c_{k}\right)\|u\|_{l^{2} X^{\sigma-2}}\|v\|_{l^{2} X^{s}}\|w\|_{l^{2} X^{s}}
$$

\section{Proof}

For the proof we need to establish trilinear estimates of the form

$$
\left\|S_{k}\left(u_{i} v_{j} w_{l}\right)\right\|_{l_{k}^{2} Y_{k}} \lesssim c_{k i j l}\left\|u_{i}\right\|_{l_{i}^{2} X_{i}}\left\|v_{j}\right\|_{l_{j}^{2} X_{j}}\left\|w_{l}\right\|_{l_{l}^{2} X_{l}} .
$$

for several different cases of frequency balances.

Case A. $|i-k| \leq 4$ and $j, l \leq k+4$. Here, we measure the output in $Y$, use $X$ for the highest frequency factor, and use $L^{\infty} L^{2}$ for the lower frequency factors. Using (2.1) we have

$$
\operatorname{LHS}(3.8) \lesssim 2^{-k / 2}\left\|S_{k}\left(u_{i} v_{j} w_{l}\right)\right\|_{Y} \lesssim 2^{-k / 2} 2^{j / 2}\left\|S_{k}\left(u_{i} v_{j} w_{l}\right)\right\|_{l_{j}^{1} L^{2} L^{2}} .
$$

By Bernstein's inequality, this yields

$$
\begin{aligned}
\operatorname{LHS}(3.8) & \lesssim 2^{-k / 2} 2^{j / 2} 2^{j d / 2}\left\|v_{j}\right\|_{l_{j}^{2} L^{\infty} L^{2}}\left\|u_{i} w_{l}\right\|_{l_{l}^{2} L^{2} L^{2}} \\
& \lesssim 2^{-k / 2} 2^{\frac{d+1}{2}} 2^{l \frac{d+1}{2}}\left\|u_{i}\right\|_{X}\left\|v_{j}\right\|_{l_{j}^{2} L^{\infty} L^{2}}\left\|w_{l}\right\|_{l_{l}^{2} L^{\infty} L^{2}},
\end{aligned}
$$


which in turn gives

$$
c_{k i j l} \lesssim 2^{-k / 2} 2^{-i / 2} 2^{l \frac{d+1}{2}} 2^{j \frac{d+1}{2}} .
$$

To prove (3.5) for this type of interaction we use (3.9) and the frequency envelopes of $u, v$, and $w$ to bound

$$
\left\|S_{k}\left(u_{i} v_{j} w_{l}\right)\right\|_{l^{2} Y^{\sigma}} \lesssim a_{i} 2^{j\left(\frac{d+3}{2}-s\right)} b_{j} 2^{l\left(\frac{d+3}{2}-s\right)} c_{l}\|u\|_{l^{2} X^{\sigma-1}}\|v\|_{l^{2} X^{s-1}}\|w\|_{l^{2} X^{s-1}},
$$

which is easily summed over $i, j, l$ in the appropriate ranges. The case where the same frequency balance holds but with the roles of $i, j$, and $l$ interchanged is similar but simpler.

Now consider (3.6). The worst case is $i \geq j \geq l$ if $\sigma \leq s-2$, respectively, $j \geq i \geq l$ if $s-2<\sigma \leq s-1$. In both cases (3.6) is weaker than (3.5).

Finally, for the estimate (3.7) we must have either $j>k-8$ or $l>k-8$, in which case (3.7) is also weaker than (3.5).

Case B. $i, j>k-4,|i-j|<4, l<i+8$. Grouping terms in the following manner,

$$
\left|\int \overline{z_{k}} u_{i} v_{j} w_{l} d x d t\right| \lesssim\left\|v_{j}\right\|_{l_{j}^{2} X_{j}}\left\|z_{k} u_{i} w_{l}\right\|_{l_{j}^{2} Y_{j}},
$$

and in view of the duality relation $l_{j}^{2} X_{j}=l_{j}^{2} Y_{j}^{*}$, from the bound (3.9) we directly obtain

$$
c_{i j k l} \lesssim 2^{-\frac{i}{2}} 2^{-\frac{j}{2}} 2^{\frac{d+1}{2} k} 2^{\frac{d+1}{2} l} .
$$

Then the estimates (3.5), (3.6), and (3.7) follow again by passing to frequency envelopes and summing over the appropriate ranges of $i, j, l$. Here, again, we have handled explicitly the worst case, and those cases where the roles of $i, j, l$ are interchanged are simpler.

We remark that in the case of (3.7) we need to deal with the additional multiplier $S_{>k-4}$. However, all our function spaces are translation invariant, and the kernel of $S_{>k-4}$ is a bounded measure. Thus it can easily be disposed of.

We shall also utilize the following bound on commutators when, for example, we decompose into functions whose Fourier transforms are supported in wedges about the coordinate axes. The proof closely resembles that of [10], though care is taken to permit $s>\frac{d+3}{2}$ rather than $s>\frac{d}{2}+2$.

PROPOSITION 3.3

We assume

$$
g^{k l}-\delta^{k l}=h^{k l}(w(t, x)),
$$

where $h(z)=O\left(|z|^{2}\right)$ near $|z|=0$. For $s>\frac{d+3}{2}$ and any multiplier $B \in S^{0}$ we have

$$
\begin{aligned}
& \left\|\nabla\left[S_{<k-4} g, B(D)\right] \nabla S_{k} u\right\|_{l_{k}^{2} Y_{k}} \\
& \quad \lesssim\|w\|_{l^{2} X^{s}}^{2}\left(1+\|w\|_{l^{2} X^{s}}\right) c\left(\|w\|_{L^{\infty}}\right)\left\|S_{k} u\right\|_{l_{k}^{2} X_{k}} .
\end{aligned}
$$


Proof

In [10, Proposition 3.2], it was shown that

$$
\nabla\left[S_{<k-4} g, B(D)\right] \nabla S_{k} u=L\left(\nabla S_{<k-4} g, \nabla S_{k} u\right),
$$

where $L$ is a translation invariant operator satisfying

$$
L(f, g)(x)=\int f(x+y) g(x+z) m(y, z) d y d z, \quad m \in L^{1} .
$$

We shall not reproduce this proof here.

Given this representation, as we are working in translation-invariant spaces, the bound (3.11) follows immediately from (3.2) and (3.5).

\section{Local energy decay}

Here we consider the main linear estimate which we shall employ in order to prove Theorem 1. This is a variant on the well-known local smoothing estimates for the linear Schrödinger equation. The metric is assumed to be a composition

$$
g^{k l}-\delta^{k l}=h^{k l}(w(t, x)),
$$

where $h(z)=O\left(|z|^{2}\right)$ near $|z|=0$. The focus, then, is on a frequency-localized linear Schrödinger equation

$$
\left(i \partial_{t}+\partial_{k} g_{<j-4}^{k l} \partial_{l}\right) u_{j}=f_{j}, \quad u_{j}(0)=u_{0 j} .
$$

In the remainder of this paper, we shall pass to this setting.

The main estimate is as follows.

\section{PROPOSITION 4.1}

Assume that the coefficients $g^{k l}$ in (4.2) satisfy (4.1) with

$$
\|w\|_{l^{2} X^{s}} \ll 1
$$

for some $s>\frac{d+3}{2}$. Let $u_{j}$ be a solution to (4.2) which is localized at frequency $2^{j}$. Then the following estimate holds:

$$
\left\|u_{j}\right\|_{l_{j}^{2} X_{j}} \lesssim\left\|u_{0 j}\right\|_{L^{2}}+\left\|f_{j}\right\|_{l_{j}^{2} Y_{j}}
$$

The proof is closely related to that given in [10] and uses a positive commutator argument. The multipliers will be first-order differential operators with smooth coefficients which are localized at frequency $\lesssim 1$; precisely, for estimating waves at frequency $2^{j}$ we will use multipliers $\mathcal{M}$ that are differential operators having the form

$$
i 2^{j} \mathcal{M}=a^{k}(x) \partial_{k}+\partial_{k} a^{k}(x)
$$

with uniform bounds on $a$ and its derivatives.

A key spot where the proof here differs from that of [10] is in the following lemma which is used to dismiss the $(g-I)$-contribution to the commutator. 


\section{LEMMA 4.2}

Let $A=\partial_{k} S_{<j-4}\left(h^{k l}(w(t, x))\right) \partial_{l}$ with $h$ as above, and let $\mathcal{M}$ be as above. Suppose that $s>\frac{d+3}{2}$. Then we have

$$
\left|\left\langle[A, \mathcal{M}] u_{j}, u_{j}\right\rangle\right| \lesssim\|w\|_{l^{2} X^{s}}^{2}\left(1+\|w\|_{l^{2} X^{s}}\right) c\left(\|w\|_{L^{\infty}}\right)\left\|u_{j}\right\|_{l_{j}^{2} X_{j}}^{2} .
$$

Proof

We note that we can abstractly write $[A, \mathcal{M}]$ as

$$
i[A, \mathcal{M}]=2^{-j}\left(\nabla(h \nabla a+a \nabla h) \nabla+\nabla h \nabla^{2} a+h \nabla^{3} a\right),
$$

where $h$ abbreviates $S_{<j-4}(h(w(t, x)))$. As the $a$-factors are bounded and low frequency, the worst term to bound is

$$
2^{-j}\left\langle a \nabla h \nabla u_{j}, \nabla u_{j}\right\rangle
$$

By duality, we only require

$$
\left\|(\nabla h) \nabla u_{j}\right\|_{l_{j}^{2} Y_{j}} \lesssim\|w\|_{l^{2} X^{s}}^{2}\left(1+\|w\|_{l^{2} X^{s}}\right) c\left(\|w\|_{L^{\infty}}\right)\left\|u_{j}\right\|_{l_{j}^{2} X_{j}},
$$

which is a consequence of (3.2) and (3.5).

We now continue the proof of the proposition. For $Q \in \mathcal{Q}_{l}, 0 \leq l \leq j$ fixed, we begin by proving that a solution $u_{j}$ to (4.2) satisfies

$$
\begin{aligned}
2^{j-l}\left\|u_{j}\right\|_{L^{2} L^{2}([0,1] \times Q)}^{2} \lesssim & \left\|u_{j}\right\|_{L^{\infty} L^{2}}^{2}+\left\|u_{j}\right\|_{X_{j}}\left\|f_{j}\right\|_{Y_{j}} \\
& +\left(2^{-j}+\|w\|_{l^{2} X^{s}}^{2}\right)\left\|u_{j}\right\|_{l_{j}^{2} X_{j}}^{2} .
\end{aligned}
$$

We start by abstracting and studying solutions to

$$
\left(D_{t}+A\right) u=f, \quad u(0)=u_{0},
$$

where $A$ is a self-adjoint operator and $D_{t}=\frac{1}{i} \partial_{t}$. First of all, we have an energytype estimate:

$$
\|u\|_{L^{\infty} L^{2}}^{2} \lesssim\left\|u_{0}\right\|_{L^{2}}^{2}+\|u\|_{X_{j}}\|g\|_{Y_{j}}+\|u\|_{L^{\infty} L^{2}}\|h\|_{L^{1} L^{2}}, \quad f=g+h
$$

(see [10, Lemma 4.2]). For a self-adjoint multiplier $\mathcal{M}$, we also have

$$
\frac{d}{d t}\langle u, \mathcal{M} u\rangle=-2 \operatorname{Im}\left\langle\left(D_{t}+A\right) u, \mathcal{M} u\right\rangle+\langle i[A, \mathcal{M}] u, u\rangle .
$$

Thus, for $u=u_{j}$ and $A=-\partial_{k} g_{<j-4}^{k l} \partial_{l}$, if we can provide a multiplier $\mathcal{M}$ so that

(1) $\|\mathcal{M} u\|_{L_{x}^{2}} \lesssim\|u\|_{L_{x}^{2}}$

(2) $\|\mathcal{M} u\|_{X} \lesssim\|u\|_{X}$

(3) $i\langle[A, \mathcal{M}] u, u\rangle \gtrsim 2^{j-\ell}\|u\|_{L^{2} L^{2}([0,1] \times Q)}^{2}-O\left(2^{-j}+\|w\|_{l^{2} X^{s}}^{2}\right)\|u\|_{l_{j}^{2} X_{j}}^{2}$,

(4.7) would follow.

We first do this when the Fourier transform of the solution $u_{j}$ is restricted to a small angle

$$
\operatorname{supp} \hat{u}_{j} \subset\left\{|\xi| \lesssim \xi_{1}\right\} \text {. }
$$


Here, we have done so about the first coordinate axis, though the modifications to obtain the same about the other axes are trivial. We take, without loss of generality due to translation invariance, $Q=\left\{\left|x_{j}\right| \leq 2^{l}: j=1, \ldots, d\right\}$, and we set $m$ to be a smooth, bounded, increasing function such that $m^{\prime}(s)=\psi^{2}(s)$ where $\psi$ is a Schwartz function localized at frequencies $\lesssim 1$, and $\psi \sim 1$ for $|s| \leq 1$. We rescale $m$ and set $m_{l}(s)=m\left(2^{-l} s\right)$. Then, we fix

$$
\mathcal{M}=\frac{1}{i 2^{j}}\left(m_{l}\left(x_{1}\right) \partial_{1}+\partial_{1} m_{l}\left(x_{1}\right)\right) .
$$

Properties (1) and (2) are immediate due to the frequency localizations of $u=u_{j}$ and $m_{l}$ as well as the boundedness of $m_{l}$. By Lemma 4.2, it suffices to consider property (3) for $A=-\Delta$. This yields

$$
i 2^{j}[-\Delta, \mathcal{M}]=-2^{-l+2} \partial_{1} \psi^{2}\left(2^{-l} x_{1}\right) \partial_{1}+O(1),
$$

and hence

$$
i 2^{j}\langle[-\Delta, \mathcal{M}] u, u\rangle=2^{-l+2}\left\|\psi\left(2^{-l} x_{1}\right) \partial_{1} u_{j}\right\|_{L^{2} L^{2}}^{2}+O\left(\left\|u_{j}\right\|_{L^{2} L^{2}}^{2}\right) .
$$

Utilizing our assumption (4.10), it follows that

$$
2^{-l} 2^{j}\left\|\psi\left(2^{-l} x_{1}\right) u_{j}\right\|_{L^{2} L^{2}}^{2} \lesssim i\left\langle[-\Delta, \mathcal{M}] u_{j}, u_{j}\right\rangle+2^{-j} O\left(\left\|u_{j}\right\|_{L^{2} L^{2}}^{2}\right),
$$

which yields (3) when combined with Lemma 4.2.

We proceed to reduce to the case when (4.10) holds. We let $\left\{\theta_{k}(\omega)\right\}_{k=1}^{d}$ be a partition of unity,

$$
\sum_{k} \theta_{k}(\omega)=1, \quad \omega \in \mathbb{S}^{d-1},
$$

where $\theta_{k}(\omega)$ is supported in a small angle about the $k$ th coordinate axis. Then, we can set $u_{j, k}=\Theta_{j, k} u_{j}$ where

$$
\mathcal{F} \Theta_{j, k} u=\theta_{k}\left(\frac{\xi}{|\xi|}\right) \sum_{j-1 \leq l \leq j+1} \phi_{l}(\xi) \hat{u}(t, \xi) .
$$

Here $\phi_{l}(\xi)$ is the Fourier multiplier associated to $S_{l}(D)$. We see that

$$
\left(D_{t}+A\right) u_{j, k}=-\Theta_{j, k} f_{j}+\left[A, \Theta_{j, k}\right] u_{j} .
$$

The operators $\Theta_{j, k}$ are bounded on $L^{2}$ by Plancherel's theorem. And as the kernel has Schwartz-type decay outside a ball of radius $2^{-j}$, it is easy to see that they are also bounded on $X$ and, via duality, $Y$. Hence, by applying $\mathcal{M}$, suitably adapted to the correct coordinate axis, to $u_{j, k}$ and summing over $k$, we obtain

$$
\begin{aligned}
& 2^{j-l}\left\|u_{j}\right\|_{L^{2} L^{2}([0,1] \times Q)}^{2} \\
& \quad \lesssim\left\|u_{j}\right\|_{L^{\infty} L^{2}}^{2}+\left\|u_{j}\right\|_{X_{j}}\left\|f_{j}\right\|_{Y_{j}} \\
& \quad+\left\|u_{j}\right\|_{l_{j}^{2} X_{j}} \sum_{k}\left\|\left[A, \Theta_{j, k}\right] u_{j}\right\|_{l_{j}^{2} Y_{j}}+\left(2^{-j}+\|w\|_{l^{2} X^{s}}^{2}\right)\left\|u_{j}\right\|_{l_{j}^{2} X_{j}}^{2} .
\end{aligned}
$$


Upon estimating the commutator term with (3.11), (4.7) follows. Taking the supremum over $Q \in \mathcal{Q}_{l}$ and subsequently over $l$ yields

$$
\left\|u_{j}\right\|_{X_{j}}^{2} \lesssim\left\|u_{j}\right\|_{L^{\infty} L^{2}}^{2}+\left\|u_{j}\right\|_{X_{j}}\left\|f_{j}\right\|_{Y_{j}}+\left(2^{-j}+\|w\|_{l^{2} X^{s}}^{2}\right)\left\|u_{j}\right\|_{l_{j}^{2} X_{j}}^{2},
$$

provided that $\|w\|_{l^{2} X^{s}}^{2}$ is bounded. By applying (4.8), we have shown

$$
\left\|u_{j}\right\|_{X_{j}}^{2} \lesssim\left\|u_{0 j}\right\|_{L^{2}}^{2}+\left\|f_{j}\right\|_{Y_{j}}^{2}+\left(2^{-j}+\|w\|_{l^{2} X^{s}}^{2}\right)\left\|u_{j}\right\|_{l_{j}^{2} X_{j}}^{2} .
$$

We now finish the proof by incorporating the summation over cubes. We let $\left\{\chi_{Q}\right\}$ denote a partition via functions which are localized to frequencies $\lesssim 1$ which are associated to cubes $Q$ of scale $M 2^{j}$. We also assume that $\left|\nabla^{k} \chi_{Q}\right| \lesssim\left(2^{j} M\right)^{-k}$, $k=1,2$. Thus,

$$
\left(D_{t}+A\right)\left(\chi_{Q} u_{j}\right)=-\chi_{Q} f_{j}+\left[A, \chi_{Q}\right] u_{j} .
$$

Applying (4.8) to $\chi_{Q} u_{j}$, we obtain

$$
\begin{aligned}
\sum_{Q}\left\|\chi_{Q} u_{j}\right\|_{L^{\infty} L^{2}}^{2} \lesssim & \left\|u_{0 j}\right\|_{L^{2}}^{2}+\left(\sum_{Q}\left\|\chi_{Q} u_{j}\right\|_{X_{j}}^{2}\right)^{1 / 2}\left(\sum_{Q}\left\|\chi_{Q} f_{j}\right\|_{Y_{j}}^{2}\right)^{1 / 2} \\
& +\sum_{Q}\left\|\left[A, \chi_{Q}\right] u_{j}\right\|_{L^{1} L^{2}}^{2} .
\end{aligned}
$$

But

$$
\sum_{Q}\left\|\left[A, \chi_{Q}\right] u_{j}\right\|_{L^{1} L^{2}}^{2} \lesssim M^{-2} \sum_{Q}\left\|\chi_{Q} u_{j}\right\|_{L^{\infty} L^{2}}^{2} \lesssim M^{-2} \sum_{Q}\left\|\chi_{Q} u_{j}\right\|_{X_{j}}^{2}
$$

For $M$ sufficiently large, we can bootstrap the commutator term of (4.13), and upon a straightforward transition to cubes of scale $2^{j}$ rather than $M 2^{j}$, we observe that

$$
\left\|u_{j}\right\|_{l_{j}^{2} L^{\infty} L^{2}}^{2} \lesssim\left\|u_{0 j}\right\|_{L^{2}}^{2}+\left\|u_{j}\right\|_{l_{j}^{2} X_{j}}\left\|f_{j}\right\|_{l_{j}^{2} Y_{j}} .
$$

We now apply (4.12) to $\chi_{Q} u_{j}$ and see that

$$
\begin{gathered}
\sum_{Q}\left\|\chi_{Q} u_{j}\right\|_{X_{j}}^{2} \lesssim \\
\left\|u_{0 j}\right\|_{L^{2}}^{2}+\sum_{Q}\left\|\chi_{Q} f_{j}\right\|_{Y_{j}}^{2}+\sum_{Q}\left\|\left[A, \chi_{Q}\right] u_{j}\right\|_{L^{1} L^{2}}^{2} \\
+\left(2^{-j}+\|w\|_{l^{2} X^{s}}^{2}\right) \sum_{Q}\left\|\chi_{Q} u_{j}\right\|_{l_{j}^{2} X_{j}}^{2} .
\end{gathered}
$$

For $M \gg 1,(4.14)$ again allows us to bootstrap the commutator term yielding

$$
M^{-d}\left\|u_{j}\right\|_{l_{j}^{2} X_{j}}^{2} \lesssim\left\|u_{0 j}\right\|_{L^{2}}^{2}+\left\|f_{j}\right\|_{l_{j}^{2} Y_{j}}^{2}+\left(2^{-j}+\|w\|_{l^{2} X^{s}}^{2}\right)\left\|u_{j}\right\|_{l_{j}^{2} X_{j}}^{2} .
$$

By (4.3), for $j$ sufficiently large (depending on $M$ ), we may absorb the last term in the right-hand side into the left. On the other hand, for the remaining bounded range of $j$, we have

$$
\|u\|_{X_{j}} \lesssim\|u\|_{L^{\infty} L^{2}}
$$

and (4.15) gives the appropriate control of the last term, which finishes the proof of (4.4). 


\section{Proof of Theorem 1}

We prove part (b) of the theorem, from which part (a) follows immediately.

We first examine the linear equation

$$
\left\{\begin{array}{l}
\left(i \partial_{t}+\partial_{k} g^{k l} \partial_{l}\right) u+V \nabla u+W u=H, \\
u(0)=u_{0}
\end{array}\right.
$$

under the assumption that

$$
g^{k l}-\delta^{k l}=h^{k l}(w(t, x)),
$$

where $h(z)=O\left(|z|^{2}\right)$ near $|z|=0$. We shall also assume that there exist smooth functions $\tilde{V}$ and $\tilde{W}$ so that

$$
V=\tilde{V}(\tilde{w}(t, x)), \quad W=\tilde{W}(v(t, x), w(t, x), \tilde{w}(t, x)),
$$

where the behavior of $\tilde{V}$ and $\tilde{W}$ near zero is given by

$$
\tilde{V}(z)=O\left(|z|^{2}\right), \quad \tilde{W}(\phi, \psi, \theta)=\phi \cdot O(|\psi|)+O\left(|\theta|^{2}\right) .
$$

Our main estimate is the following.

\section{PROPOSITION 5.1}

(a) Assume that the metric $g$ and potentials $V$ and $W$ are as above and that they satisfy

$$
\|w\|_{l^{2} X^{s}} \ll 1, \quad\|\tilde{w}\|_{l^{2} X^{s-1}} \ll 1, \quad\|v\|_{l^{2} X^{s-2}} \ll 1, \quad s>\frac{d+3}{2} .
$$

Then (5.1) is well-posed for initial data $u_{0} \in H^{\sigma}$ with $0 \leq \sigma \leq s-1$, and we have the estimate

$$
\|u\|_{l^{2} X^{\sigma}} \lesssim\left\|u_{0}\right\|_{H^{\sigma}}+\|H\|_{l^{2} Y^{\sigma}} .
$$

(b) Assume in addition that $W=0$. Then (5.1) is well-posed for initial data $u_{0} \in H^{\sigma}$ with $0 \leq \sigma \leq s$, and the estimate (5.2) holds.

Proof

The existence part follows in a standard manner by approximating the metric, potentials, and data by Schwartz functions and passing to the limit on a subsequence, provided that we have the uniform bound (5.2). Hence the remainder of the proof is devoted to establishing (5.2).

For $u$ solving (5.1), we see that $u_{j}$ solves

$$
\left\{\begin{array}{l}
\left(i \partial_{t}+\partial_{k} g_{<j-4}^{k l} \partial_{l}\right) u_{j}=G_{j}+H_{j}, \\
u_{j}(0)=u_{0 j}
\end{array}\right.
$$

where

$$
G_{j}=-S_{j} \partial_{k} g_{>j-4}^{k l} \partial_{l} u-\left[S_{j}, \partial_{k} g_{<j-4}^{k l} \partial_{l}\right] u-S_{j} V \nabla u-S_{j} W u
$$


If we apply Proposition 4.1 to each of these equations, we see that

$$
\|u\|_{l^{2} X^{\sigma}}^{2} \lesssim\left\|u_{0}\right\|_{H^{\sigma}}^{2}+\|H\|_{l^{2} Y^{\sigma}}^{2}+\sum_{j}\left\|G_{j}\right\|_{l^{2} Y^{\sigma}}^{2}
$$

We claim that

$$
\sum_{j}\left\|G_{j}\right\|_{l^{2} Y^{\sigma}}^{2} \lesssim\|u\|_{l^{2} X^{\sigma}}^{2}\left(\|w\|_{l^{2} X^{s}}^{4}+\|\tilde{w}\|_{l^{2} X^{s-1}}^{4}+\|v\|_{l^{2} X^{s-2}}^{4}\right) .
$$

Indeed, for the first term in $G_{j}$ we apply (3.2), (3.5), and (3.7). The bound for the second term in $G_{j}$ follows from (3.11). The bounds for the last two terms of $G_{j}$ use (3.5) and (3.6), respectively, in conjunction with (3.2). The differences in the range of permissible $\sigma$ in (3.5) versus (3.6) accounts precisely for the difference in parts (a) and (b) of this proposition.

We now set up an iteration to solve (1.2). We set $u^{(0)} \equiv 0$ and recursively define $u^{(n+1)}$ to be the solution to

$$
\left\{\begin{array}{l}
\left(i \partial_{t}+\partial_{j} g^{j k}\left(u^{(n)}\right) \partial_{k}\right) u^{(n+1)}=F\left(u^{(n)}, \nabla u^{(n)}\right), \\
u^{(n+1)}(0, x)=u_{0}(x) .
\end{array}\right.
$$

As $F(y, z)=O\left(|y|^{3}+|z|^{3}\right)$ near the origin, it follows from (3.2) with $\sigma=s-1$ and (3.5) with $\sigma=s$ that

$$
\left\|F\left(u^{(n)}, \nabla u^{(n)}\right)\right\|_{l^{2} Y^{s}} \lesssim\left\|u^{(n)}\right\|_{l^{2} X^{s}}^{3}
$$

provided that $s>\frac{d+3}{2}$ and $\left\|u^{(n)}\right\|_{l^{2} X^{s}}=O(1)$. Using this in each application of Proposition 5.1, we see, via induction, that

$$
\left\|u^{(n)}\right\|_{l^{2} X^{s}} \lesssim\left\|u_{0}\right\|_{H^{s}}
$$

for each $n$ provided that $\left\|u_{0}\right\|_{H^{s}}$ is sufficiently small.

We now seek to show that the iteration converges in $l^{2} X^{s-1}$. To this end, we note that $v^{(n+1)}=u^{(n+1)}-u^{(n)}$ solves

$$
\left\{\begin{array}{l}
\left(i \partial_{t}+\partial_{j} g^{j k}\left(u^{(n)}\right) \partial_{k}\right) v^{(n+1)}=V_{n} \nabla v^{(n)}+W_{n} v^{(n)} \\
v^{(n+1)}(0, x)=0
\end{array}\right.
$$

where

$$
\begin{aligned}
V_{n} & =V_{n}\left(u^{(n)}, \nabla u^{(n)}, u^{(n-1)}, \nabla u^{(n-1)}\right), \\
W_{n} & =h_{1}\left(u^{(n)}, \nabla u^{(n)}, u^{(n-1)}, \nabla u^{(n-1)}\right)+h_{2}\left(u^{(n)}, u^{(n-1)}\right) \nabla^{2} u^{(n)} .
\end{aligned}
$$

Here $V_{n}(z)=O\left(|z|^{2}\right), h_{1}(z)=O\left(|z|^{2}\right)$, and $h_{2}(z)=O(|z|)$ near $|z|=0$. By Proposition 5.1, we have

$$
\left\|v^{(n+1)}\right\|_{l^{2} X^{s-1}} \lesssim\left\|V_{n} \nabla v^{(n)}\right\|_{l^{2} Y^{s-1}}+\left\|W_{n} v^{(n)}\right\|_{l^{2} Y^{s-1}} .
$$

By (3.5) and (3.6) with $\sigma=s-1$, we have

$$
\begin{aligned}
\left\|V_{n} \nabla v^{(n)}\right\|_{l^{2} Y^{s-1}} & \lesssim\left(\left\|u^{(n)}\right\|_{l^{2} X^{s}}+\left\|u^{(n-1)}\right\|_{l^{2} X^{s}}\right)^{2}\left\|v^{(n)}\right\|_{l^{2} X^{s-1}}, \\
\left\|W_{n} v^{(n)}\right\|_{l^{2} Y^{s-1}} & \lesssim\left(\left\|u^{(n)}\right\|_{l^{2} X^{s}}+\left\|u^{(n-1)}\right\|_{l^{2} X^{s}}\right)^{2}\left\|v^{(n)}\right\|_{l^{2} X^{s-1}}
\end{aligned}
$$


Thus, it follows from (5.4) that

$$
\left\|v^{(n+1)}\right\|_{l^{2} X^{s-1}} \ll\left\|v^{(n)}\right\|_{l^{2} X^{s-1}},
$$

which implies that the iteration converges in $l^{2} X^{s-1}$ to a function $u$ satisfying

$$
\|u\|_{l^{2} X^{s}} \lesssim\left\|u_{0}\right\|_{H^{s}}
$$

This establishes the existence of a solution.

We next consider the question of uniqueness. For two solutions $u^{(1)}$ and $u^{(2)}$ to (1.2), we consider $v=u^{(2)}-u^{(1)}$. Here, $v$ solves

$$
\left\{\begin{array}{l}
\left(i \partial_{t}+\partial_{j} g^{j k}\left(u^{(2)}\right) \partial_{k}\right) v=V \nabla v+W v \\
v(0, x)=u^{(2)}(0, x)-u^{(1)}(0, x)
\end{array}\right.
$$

where

$$
\begin{aligned}
V & =V\left(u^{(1)}, \nabla u^{(1)}, u^{(2)}, \nabla u^{(2)}\right), \\
W_{n} & =h_{1}\left(u^{(2)}, \nabla u^{(2)}, u^{(1)}, \nabla u^{(1)}\right)+h_{2}\left(u^{(2)}, u^{(1)}\right) \nabla^{2} u^{(1)} .
\end{aligned}
$$

By Proposition 5.1, we have

$$
\left\|u^{(2)}-u^{(1)}\right\|_{l^{2} X^{s-1}} \lesssim\left\|u^{(2)}(0)-u^{(1)}(0)\right\|_{H^{s-1}},
$$

from which uniqueness follows.

To show continuity of the map $u_{0} \rightarrow u$ from $H^{s} \rightarrow l^{2} X^{s}$, we seek to strengthen the preceding argument. To do so, we shall use frequency envelopes. Indeed, we first seek to strengthen (5.7) by showing that a frequency envelope for the data is also a frequency envelope for the solution.

\section{PROPOSITION 5.2}

Let $s>\frac{d+3}{2}$, and let $u \in l^{2} X^{s}$ be a small data solution to (1.2), which satisfies (5.7). If $\left\{a_{j}\right\}$ is an admissible frequency envelope for the initial data $u_{0}$ in $H^{s}$, then $\left\{a_{j}\right\}$ is also a frequency envelope for $u$ in $l^{2} X^{s}$.

Proof

We set

$$
b_{j}=2^{-\delta j}+\|u\|_{l^{2} X^{s}}^{-1} \max _{k} 2^{-\delta|j-k|}\left\|S_{k} u\right\|_{l^{2} X^{s}}
$$

and note that $\left\{b_{j}\right\}$ is a frequency envelope for $u$ in $l^{2} X^{s}$. We note that $u_{j}$ solves

$$
\left\{\begin{array}{l}
\left(i \partial_{t}+\partial_{k} g_{<j-4}^{k l} \partial_{l}\right) u_{j} \\
\quad=S_{j} F(u, \nabla u)-S_{j} \partial_{k} g_{>j-4}^{k l} \partial_{l} u-\left[S_{j}, \partial_{k} g_{<j-4}^{k l} \partial_{l}\right] u \\
u_{j}(0)=\left(u_{0}\right)_{j} .
\end{array}\right.
$$

Labeling the right-hand side of the equation above $f_{j}$, we apply Proposition 5.1 and obtain

$$
\left\|S_{j} u\right\|_{l^{2} X^{s}} \lesssim a_{j}\left\|u_{0}\right\|_{H^{s}}+\left\|f_{j}\right\|_{l^{2} Y^{s}} .
$$


We bound $f_{j}$ in a manner that is akin to the above. This yields

$$
\left\|f_{j}\right\|_{l^{2} Y^{s}} \lesssim b_{j}\|u\|_{l^{2} X^{s}}^{3} c\left(\|u\|_{l^{2} X^{s}}\right) .
$$

Indeed, provided that $s>\frac{d+3}{2}$, we can apply (3.2) $(\sigma=s-1)$ and (3.5) $(\sigma=s)$ to bound the first term. For the second term we use (3.2) $(\sigma=s),(3.5)$, and (3.7). For the last term in $f_{j}$, we apply (3.11).

Applying (5.13) in (5.12) we obtain

$$
\left\|S_{j} u\right\|_{l^{2} X^{s}} \lesssim a_{j}\left\|u_{0}\right\|_{H^{s}}+b_{j}\|u\|_{l^{2} X^{s}}^{3} c\left(\|u\|_{l^{2} X^{s}}\right),
$$

which yields

$$
b_{j} \lesssim a_{j}\left\|u_{0}\right\|_{H^{s}}\|u\|_{l^{2} X^{s}}^{-1}+b_{j}\|u\|_{l^{2} X^{s}}^{2} c\left(\|u\|_{l^{2} X^{s}}\right) .
$$

Using the smallness of $\|u\|_{l^{2} X^{s}}$, we can bootstrap the second term in the righthand side. Moreover, by the definition of $l^{2} X^{s}$, we have $\left\|u_{0}\right\|_{H^{s}} \lesssim\|u\|_{l^{2} X^{s}}$. Thus, the above implies $b_{j} \lesssim a_{j}$ as desired.

We now proceed to show that the map $H^{s} \rightarrow l^{2} X^{s}$ given by $u_{0} \mapsto u \neq 0$ is continuous. (The case $u_{0}=0$ follows immediately from (5.7).) Let $\left\{u_{0}^{(n)}\right\} \subset H^{s}$ be a sequence which converges to $u_{0}$ in $H^{s}$, and let $\left\{a_{j}^{(n)}\right\}$ and $\left\{a_{j}\right\}$ denote their respective frequency envelopes defined via (2.3). It follows, thus, that $a_{j}^{(n)} \rightarrow a_{j}$ in $l^{2}$. For any $\varepsilon>0$, there is an $N_{\varepsilon}$ such that

$$
\left\|a_{j}^{(n)}\right\|_{l^{2}\left(j>N_{\varepsilon}\right)} \leq \varepsilon, \quad\left\|a_{j}\right\|_{l^{2}\left(j>N_{\varepsilon}\right)} \leq \varepsilon
$$

uniformly in $n$. The preceding proposition then yields

$$
\begin{aligned}
& \left\|u_{>N_{\varepsilon}}^{(n)}\right\|_{l^{2} X^{s}} \leq \varepsilon\left\|u^{(n)}\right\|_{l^{2} X^{s}} \leq C \varepsilon\left\|u_{0}^{(n)}\right\|_{H^{s}}, \\
& \left\|u_{>N_{\varepsilon}}\right\|_{l^{2} X^{s}} \leq \varepsilon\|u\|_{l^{2} X^{s}} \leq C \varepsilon\left\|u_{0}\right\|_{H^{s}},
\end{aligned}
$$

where in the last step we applied (5.7).

To compare $u^{(n)}$, where $u^{(n)}$ is the solution to (1.2) with datum $u_{0}^{(n)}$, to $u$, we use (5.14) for the high frequencies and (5.9) for the low frequencies. Indeed,

$$
\begin{aligned}
\left\|u^{(n)}-u\right\|_{l^{2} X^{s}} & \lesssim\left\|S_{<N_{\varepsilon}}\left(u^{(n)}-u\right)\right\|_{l^{2} X^{s}}+\left\|u_{>N_{\varepsilon}}^{(n)}\right\|_{l^{2} X^{s}}+\left\|u_{>N_{\varepsilon}}\right\|_{l^{2} X^{s}} \\
& \lesssim 2^{N_{\varepsilon}}\left\|S_{<N_{\varepsilon}}\left(u^{(n)}-u\right)\right\|_{l^{2} X^{s-1}}+\varepsilon\left\|u_{0}^{(n)}\right\|_{H^{s}}+\varepsilon\left\|u_{0}\right\|_{H^{s}} \\
& \lesssim 2^{N_{\varepsilon}}\left\|S_{<N_{\varepsilon}}\left(u_{0}^{(n)}-u_{0}\right)\right\|_{H^{s-1}}+\varepsilon\left\|u_{0}^{(n)}\right\|_{H^{s}}+\varepsilon\left\|u_{0}\right\|_{H^{s}} .
\end{aligned}
$$

Letting $n \rightarrow \infty$ yields

$$
\limsup _{n \rightarrow \infty}\left\|u^{(n)}-u\right\|_{l^{2} X^{s}} \lesssim \varepsilon\left\|u_{0}\right\|_{H^{s}}
$$

and subsequently letting $\varepsilon \rightarrow 0$ yields the desired result.

We finish by showing the analogue of (5.7) for higher frequencies:

$$
\|u\|_{l^{2} X^{\sigma}} \lesssim\left\|u_{0}\right\|_{H^{\sigma}}, \quad \sigma \geq s,
$$

assuming that $u_{0} \in H^{\sigma}$. Differentiating the original equation yields 


$$
\begin{aligned}
\left(i \partial_{t}+\partial_{j} g^{j k}(u) \partial_{k}\right)\left(\partial_{l} u\right)= & -\left(g^{j k}\right)^{\prime}(u)\left(\partial_{j} \partial_{l} u \partial_{k} u+\partial_{l} u \partial_{j} \partial_{k} u\right) \\
& -\left(g^{j k}\right)^{\prime \prime}(u)\left(\partial_{j} u \partial_{l} u \partial_{k} u\right)+\left(\nabla_{z_{1}} F\right)(u, \nabla u) \cdot \nabla \partial_{l} u \\
& +F_{z_{0}}(u, \nabla u) \partial_{l} u
\end{aligned}
$$

For $v_{1}=\nabla u$, we have

$$
\left(i \partial_{t}+\partial_{j} g^{j k}(u) \partial_{k}\right) v_{1}=G(u, \nabla u) \nabla v_{1}+F_{1}(u, \nabla u),
$$

where $G(z)=O\left(|z|^{2}\right)$ and $F_{1}(z)=O\left(|z|^{3}\right)$ near $z=0$. By (3.2) and (3.5), we have

$$
\left\|G(u, \nabla u) \nabla v_{1}\right\|_{l^{2} Y^{s}} \lesssim\|u\|_{l^{2} X^{s}}^{2}\left\|v_{1}\right\|_{l^{2} X^{s}}, \quad\left\|F_{1}(u, \nabla u)\right\|_{l^{2} Y^{s}} \lesssim\|u\|_{l^{2} X^{s}}^{3}
$$

And by Proposition 5.1,

$$
\left\|v_{1}\right\|_{l^{2} X^{s}} \lesssim\left\|v_{1}(0)\right\|_{H^{s}}+\|u\|_{l^{2} X^{s}}^{3}
$$

and thus,

$$
\|u\|_{l^{2} X^{s+1}} \lesssim\|u(0)\|_{H^{s+1}}+\|u\|_{l^{2} X^{s}}^{3}
$$

Letting $v_{n}=\nabla^{n} u$, we see that $v_{n}$ solves

$$
\left(i \partial_{t}+\partial_{j} g^{j k}(u) \partial_{k}\right) v_{n}=G(u, \nabla u) \nabla v_{n}+F_{n}\left(u, \nabla u, \ldots, \nabla^{n} u\right)
$$

with $G$ as above. Arguing inductively, we obtain

$$
\left\|v_{n}\right\|_{l^{2} X^{s}} \lesssim\left\|v_{n}(0)\right\|_{H^{s}}+\|u\|_{l^{2} X^{s+n-1}}^{3}
$$

which gives

$$
\|u\|_{l^{2} X^{s+n}} \lesssim\|u(0)\|_{H^{s+n}}+\|u\|_{l^{2} X^{s+n-1}}^{3},
$$

from which the desired conclusion follows.

Acknowledgments. The authors wish to thank the anonymous reviewers for a careful reading of the draft.

\section{References}

[1] I. Bejenaru and D. Tataru, Large data local solutions for the derivative NLS equation, J. Eur. Math. Soc. (JEMS) 10 (2008), 957-985. MR 2443925. DOI 10.4171/JEMS/136.

[2] W. Ichinose, On $L^{2}$ well-posedness of the Cauchy problem for Schrödinger type equations on the Riemannian manifold and Maslov theory, Duke Math. J. 56 (1988), 549-588. MR 0948533. DOI 10.1215/S0012-7094-88-05623-2.

[3] C. Kenig, G. Ponce, C. Rolvung, and L. Vega, Variable coefficient Schrödinger flows for ultrahyperbolic operators, Adv. Math. 196 (2005), 373-486.

MR 2166312. DOI 10.1016/j.aim.2004.02.002.

[4] , The general quasilinear ultrahyperbolic Schrödinger equation, Adv. Math. 206 (2006), 402-433. MR 2263709. DOI 10.1016/j.aim.2005.09.005. 
[5] C. E. Kenig, G. Ponce, and L. Vega, Small solutions to nonlinear Schrödinger equations, Ann. Inst. H. Poincaré Anal. Non Linéaire 10 (1993), 255-288. MR 1230709.

[6] _ Smoothing effects and local existence theory for the generalized nonlinear Schrödinger equations, Invent. Math. 134 (1998), 489-545. MR 1660933. DOI 10.1007/s002220050272.

[7] - The Cauchy problem for quasi-linear Schrödinger equations, Invent. Math. 158 (2004), 343-388. MR 2096797. DOI 10.1007/s00222-004-0373-4.

[8] F. Linares and G. Ponce, Introduction to Nonlinear Dispersive Equations, Universitext, Springer, New York, 2009. MR 2492151.

[9] J. Marzuola, J. Metcalfe, and D. Tataru, Wave packet parametrices for evolutions governed by PDO's with rough symbols, Proc. Amer. Math. Soc. 136 (2008), 597-604. MR 2358501. DOI 10.1090/S0002-9939-07-09027-2.

[10] , Quasilinear Schrödinger equations, I: Small data and quadratic interactions, Adv. Math. 231 (2012), 1151-1172. MR 2955206.

DOI 10.1016/j.aim.2012.06.010.

[11] S. Mizohata, Some remarks on the Cauchy problem, J. Math. Kyoto Univ. 1 (1961/1962), 109-127. MR 0170112.

[12] - On some Schrödinger type equations, Japan Proc. Acad. Ser. A Math. 57 (1981), 81-84.

[13] - On the Cauchy Problem, Notes and Reports in Mathematics in Science and Engineering 3, Academic Press, Orlando, 1985. MR 0860041.

Marzuola: Department of Mathematics, University of North Carolina-Chapel Hill, Phillips Hall, Chapel Hill, North Carolina 27599-3250, USA; marzuola@email.unc.edu

Metcalfe: Department of Mathematics, University of North Carolina-Chapel Hill, Phillips Hall, Chapel Hill, North Carolina 27599-3250, USA; metcalfe@email.unc.edu

Tataru: Mathematics Department, University of California, Evans Hall, Berkeley, California 94720, USA; tataru@math.berkeley.edu 